\title{
Covid-19 mimicking breast cancer progression
}

\author{
Joanna Kufel-Grabowska ${ }^{1,2}$, Iwona Mozer-Lisewska ${ }^{3}$, Witold Cholewiński1,4, \\ Mikołaj Bartoszkiewicz ${ }^{5,6}$, Maria Litwiniuk ${ }^{6,7}$
}

\author{
'Department of Electroradiology, Poznan University of Medical Sciences, Poznan, Poland \\ ${ }^{2}$ Department of Chemotherapy, University Hospital of Lord's Transfiguration, Poznan Poland \\ ${ }^{3}$ Department of Infectious Diseases, Poznan University of Medical Sciences, Poznan, Poland \\ ${ }^{4}$ Nuclear Medicine Department, The Greater Poland Cancer Centre, Poznan, Poland \\ ${ }^{5}$ Department of Immunobiology, Poznan University of Medical Sciences, Poznan, Poland \\ ${ }^{6}$ Department of Chemotherapy, The Greater Poland Cancer Center, Poznan, Poland \\ ${ }^{7}$ Department of Cancer Pathology and Prevention, Poznan University of Medical Sciences, Poznan, Poland
}

\section{Abstract}

In February 2019, a 73-year-old woman reported with a tumour of the right breast and enlarged lymph nodes in the right axilla. A core-needle biopsy of the tumour was performed, which confirmed the diagnosis of invasive breast carcinoma NST G2, oestrogen-dependent, HER2-negative; ki 67 was $2 \%$. Imaging showed metastases in the lungs and liver, and numerous osteolytic lesions in the bones. The patient was qualified for ribociclib therapy in combination with letrozole and zoledronic acid, and she received palliative irradiation of Th6-Th8 and Th12-L2. The treatment stabilized the cancer and the patient's general condition by February 2020. In a PET scan performed at that time previously unobserved lymph nodes in the mediastinum and liver recess were described; the lesions were ambiguous. In July 2020, there was progression of metastatic changes in the lymph nodes. CDK4/6 inhibitor and letrozole were discontinued, tamoxifen was included, and zoledronic acid therapy was continued. After the treatment change there was a rapid deterioration of the patient's general condition; she patient was drowsy and confused. The spread of cancer to the central nervous system (CNS) was suspected. The patient's husband and daughter (physicians) were diagnosed with a coronavirus infection. The patient's test was also positive; thus, she was referred to the infectious diseases ward. The dominant symptom was progressive weakness, followed by neurological symptoms: confusion, dizziness, and disinhibition reaction. Her general condition did not allow CNS imaging diagnostics. During hospitalization the patient was treated symptomatically, but her general condition did not improve. The patient died after 16 days of hospitalization.

\section{Address for correspondence:}

Mikołaj Bartoszkiewicz, Department of Immunobiology, Poznan University of Medical

Sciences, 8 Rokietnicka St., 60-806, Poznan, Poland, phone: +48 6185476 53,

e-mail: m.bartoszkiewicz@ump.edu.pl

\section{INTRODUCION}

In February 2019, 73-year-old woman reported with right breast and enlarged lymph nodes in right axilla. Core-needle biopsy of the tumour was performed, and it confirmed the diagnosis of invasive breast carcer NST G2, oestrogen-dependent, HER2-negative; ki 67 was 2\%. Imaging showed metastases in the lungs and liver, and numerous osteolytic lesions in the bones with vertebral body fractures (Th6-Th12) (Fig. $1 \mathrm{~A}-\mathrm{C})$. The family history was negative; the patient had been treated for many years due to hypertension and ascending aortic aneurysm. A few years earlier she had not consented to surgical treatment of the aneurysm; she was under constant cardiac care, and her general condition was stable (ECOG 1).

\section{MATERIAL AND METHODS}

The patient was qualified for ribociclib therapy in combination with letrozole and zoledronic acid. She received palliative irradiation of Th6-Th8 and Th12-L2, which alleviated her pain; painkillers were also included. The treatment stabilized the cancer and her general condition by February 2020. In a PET scan performed at the time previously unobserved lymph nodes in the mediastinum and liver recess were described; the lesions were ambiguous (Fig. 1 D, F). In July 2020, progression was seen in suspicious lymph nodes (Fig. $1 \mathrm{G}-\mathrm{I}$ ). The patient reported progressive weakness and deterioration of appetite. CDK4/6 inhibitor and letrozole were discontinued, tamoxifen was included, and zoledronic acid therapy was continued. 

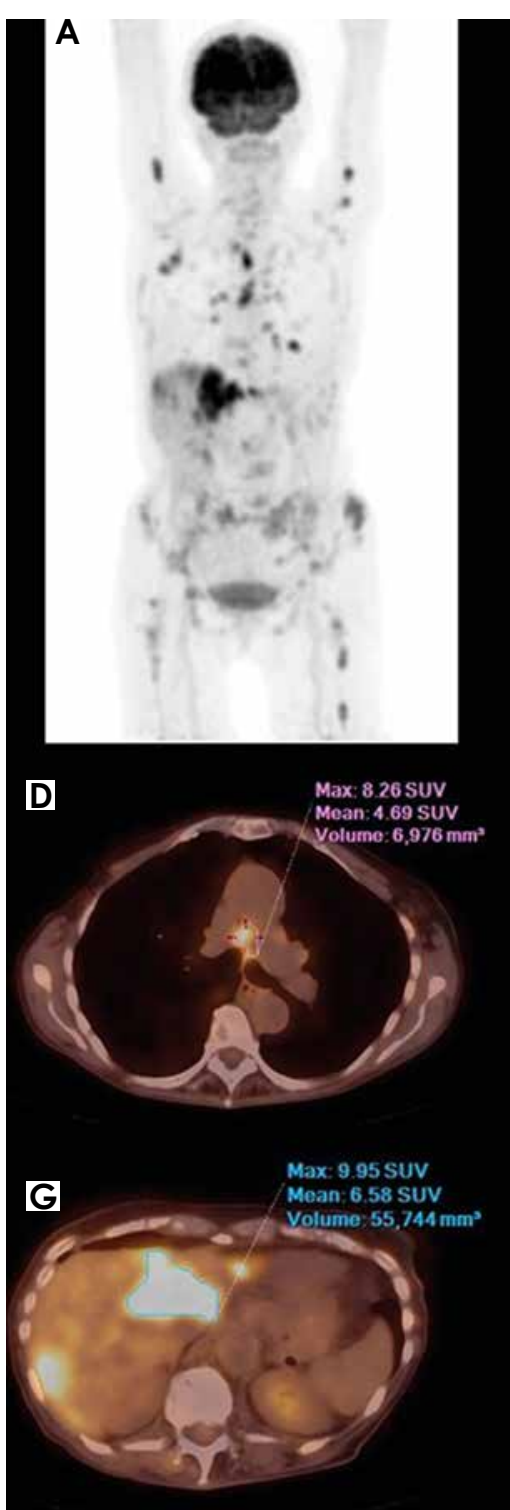

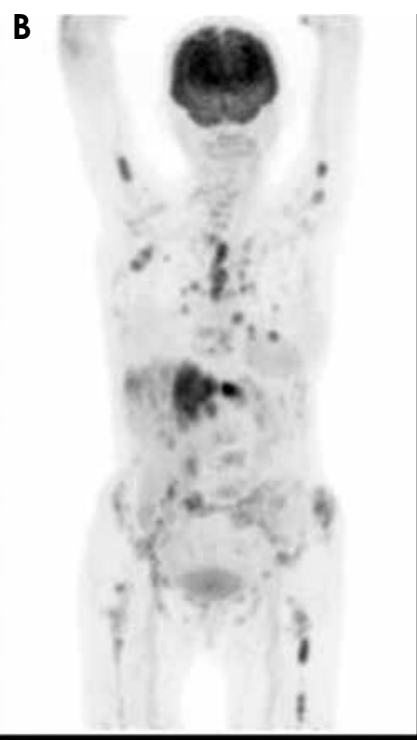

E

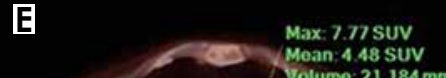

C

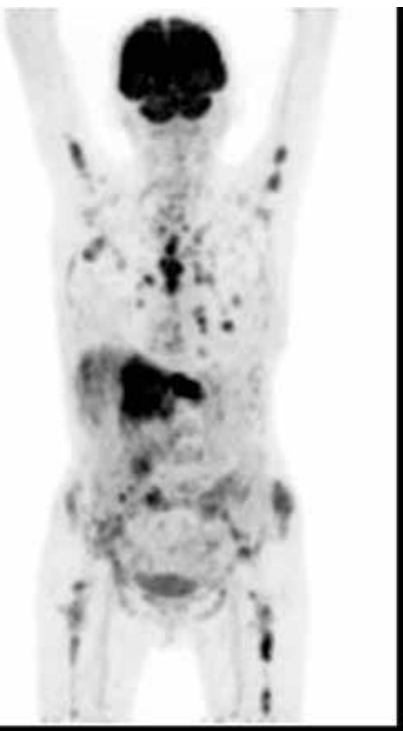

\section{F}

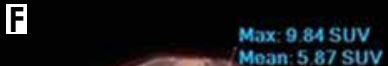

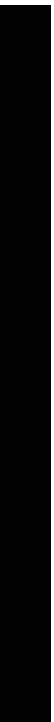
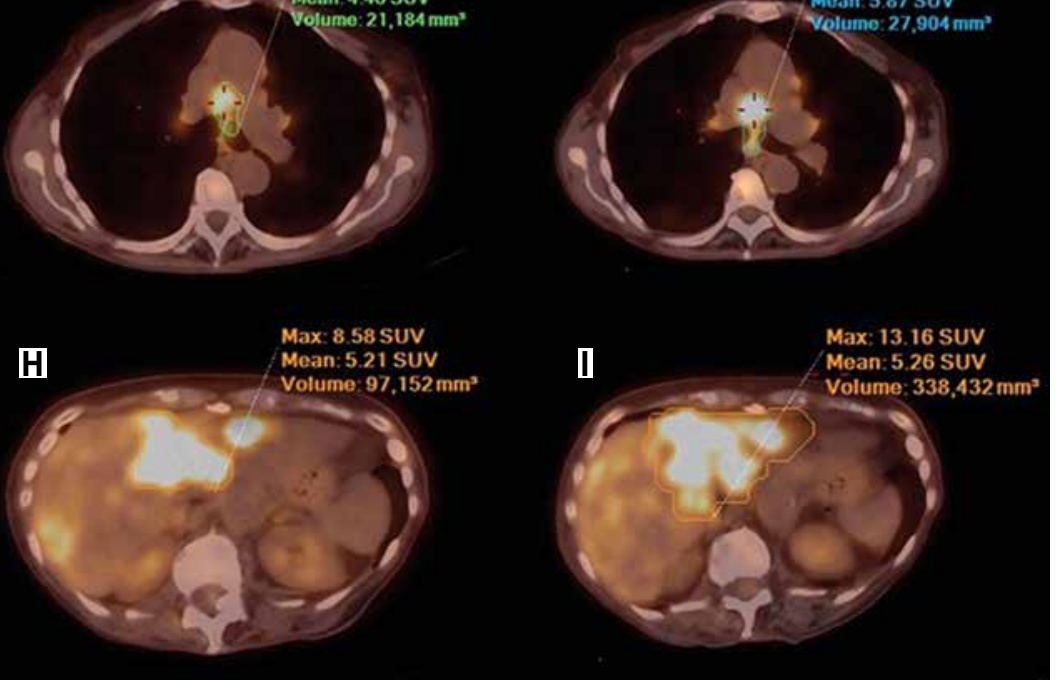

Fig. 1. The patient's 18F-FDG-PET/CT scans performed in November 2019 (A-C), February 2020 (D, F), and June 2020 right column). Rows represent whole body MIP picture (upper row), mediastinal nodes (middle row), and metastases in the left lobe of the liver (G-I). Multiple metastatic lesions are observed throughout the whole-body scan with morphologic and metabolic progression over time seen both in lesion volumes and metabolic activity measured as SUV values

\section{RESULTS}

After the treatment change there was a rapid deterioration of the patient's general condition; she was drowsy and confused. The spread of cancer to the central nervous system (CNS) was suspected.

The patient's husband and daughter (physicians) were diagnosed with coronavirus infection. Husband had a fever and cough, and the daughter was completely asymptomatic. The patient's test was also positive; thus, she was referred to the infectious diseases ward. The dominant symptom was progressive weakness, followed by neurological abnormalities: confusion, dizziness, and disinhibition reaction. Her general condition did not allow CNS imaging diagnostics. During hospitalization the patient was treated symptomatically, but her general condition did not improve. The patient died after 16 days of hospitalization. Probably the patient's death was due to heart failure, but no autopsy was performed.

\section{DISCUSSION}

Breast cancer is the most common cancer in women worldwide. Thanks to modern therapies, metastatic breast cancer can be treated as a chronic disease [1].

Literature data suggest that the mortality of coronavirus-infected breast cancer patients is more dependent on coexisting diseases and age ( $>70$ years of age, hypertension) than the anticancer treatment [2]. The 
diagnosis of SARS-CoV2 infection in patients without classical symptoms is challenging, which may delay treatment. In the course of coronavirus infection, neurological symptoms may appear, the most common being anosmia and ageusia. Less frequent are strokes, Guillain-Barre syndrome, and encephalitis. According to the literature, encephalopathy appears in $7-69 \%$ of patients, and the incidence increases with the severity of coronavirus infection [3].

\section{CONCLUSIONS}

In the presented case, the symptoms of SARSCoV2 infection mimicked metastatic spread to the CNS. Confirmed SARS-CoV2 infections in the family led to the diagnosis of this infection in the patient.

Special attention should be paid to cancer patients with comorbidities, especially during anticancer therapy, because symptoms of coronavirus infection may be non-specific, and an undiscovered infection can be fatal.

The authors declare no conflict of interest.

\section{REFERNCES}

1. Delaloge S, Ezzalfani M, Dieras V, et al. Evolution of overall survival according to year of diagnosis (2008-2014) and subtypes, among 16703 metastatic breast cancer (MBC) patients included in the real-life "ESME" cohort. J Clin Oncol 2017; 35: 1078-1078.

2. Vuagnat $P$, Frelaut M, Ramtohul T, et al. COVID-19 in breast cancer patients: a cohort at the Institut Curie hospitals in the Paris area. Breast Cancer Res 2020; 22: 55.

3. Ellul MA, Benjamin L, Singh B, et al. Neurological associations of COVID-19. Lancet Neurol 2020; 19: 767-783. 